www.lpr-journal.org

LASER \& PHOTONICS

REVIEWS 
Abstract A chiral arrangement of molecular nanoemitters is shown to support delocalised exciton states whose spontaneous decay can generate optical vortex radiation. In contrast to techniques in which phase modification is imposed upon conventional optical beams, this exciton method enables radiation with a helical wave-front to be produced directly. To achieve this end, a number of important polarisation and symmetry-based criteria need to be satisfied. It emerges that the phase structure of the optical field produced by degenerate excitons in a propeller-shaped array can exhibit precisely the sought character of an optical vortex - one with unit topological charge. Practical considerations for the further development of this technique are discussed, and potential new applications are identified.

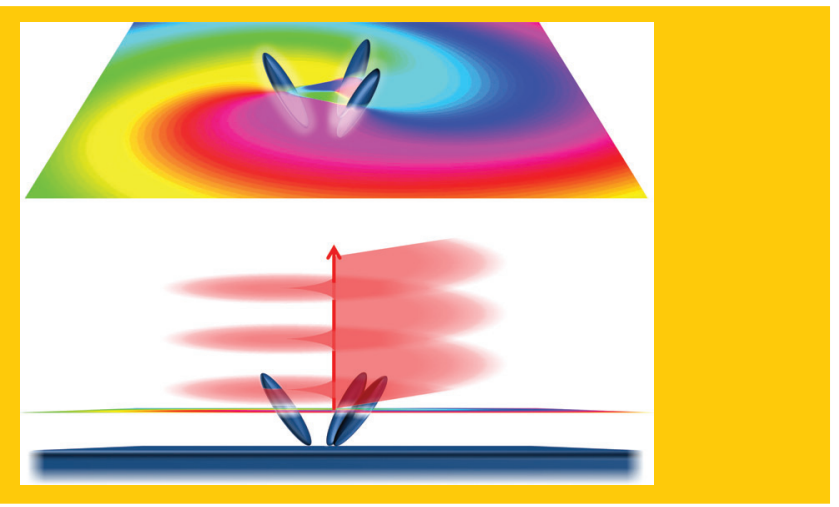

\title{
Chiral nanoemitter array: A launchpad for optical vortices
}

\author{
Matt M. Coles, Mathew D. Williams, Kamel Saadi, David S. Bradshaw, and David L. Andrews*
}

\section{Introduction}

Some of the most ingenious advances to emerge from the field of optics centre upon the concept that light can convey orbital angular momentum [1], distinct and separable from spin angular momentum [2] associated with circular polarisations. The discovery has not only led to a reappraisal of fundamental theory; it now promises a wide range of new applications, extending from quantum information to nanoscale motor action [3-8]. With suitable optical elements it proves relatively simple to produce an optical vortex, or twisted beam, whose helical wave-front conveys orbital angular momentum. Typically, passing light with a conventional beam profile, through a phase mask or a bifurcated diffraction grating, produces a Laguerre-Gaussian beam. Such beams have an azimuthal phase, with topological charge of integer $l$ corresponding to an orbital angular momentum of $l \hbar$ per photon.

Generally, it has been considered unfeasible to directly emit photons with orbital angular momentum. Fundamental theory shows that dipole-allowed spectroscopic transitions within individual atoms and molecules do not engage with, or produce, orbital forms of optical angular momentum [9]. Where higher multipole orders are involved, atomic centreof-mass motions need to be harnessed [10], associated with orbital motion around the beam [11]; however, such effects are extremely weak and cannot arise in the condensed phase. Moreover, it has been shown that photons released in any multipolar transition fail to communicate orbital angular momentum to any conventional detector [12]. However, these obstacles are not insurmountable. As we demonstrate, it is possible, by exploiting the non-local nature of the photon itself, to achieve the direct emission of light with orbital angular momentum. The key is to exploit specific forms of electronic state with a symmetry that can only be supported by molecules.

In some of the earliest literature on optical vortices it was remarked that to tailor an optical wave-front with the necessary twist generally demands material inhomogeneity [1]. For the direct generation of vortex light there are more demanding conditions to be satisfied, involving not only the source profile but also its electronic structure. In particular, it emerges that these conditions can be fulfilled with molecular nanoarrays, constructed to support a specific form of exciton based on delocalised excitation. Emitters grouped in ring-like arrays will support certain delocalised electronic states (excitons) that occur in degenerate pairs of left- and right-handed circularity. The release of energy from such an exciton can produce the emission of a photon with the sought helical wave-front - once a few additional conditions are satisfied.

The process whereby individual photons are emitted with azimuthal phase is of fundamental interest, and the mapping of delocalised electronic excitation to a transverse optical field structure is also novel. Furthermore, the following findings represent a contribution to the on-going debate over the amount of information potentially communicable by a single photon [13-19] - a consideration linking with the aspiration for a greatly increased speed and data content of free-space data transmission [20].

\section{Chiral exciton formation}

The exploitation of phase relationships between the quantum amplitudes relating to emission at different sites is a 
familiar concept in parametric nonlinear optics; it is responsible for the laser-like character of optical harmonic generation, for example. With the exception of superradiance, such phase relationships do not usually arise in spontaneous emission. However, by adopting an array with a suitable geometric configuration, it is possible to engineer phase relationships of an equivalent form, correlated with the emitter positions. The present application demands a phase relationship that matches the angular positions of the associated emitters about a given axis, the latter serving to define a director for the propagation of a suitable optical mode. In this case, the azimuthal character of this mode is exactly that of an $l=1$ optical vortex.

Excitons can exactly satisfy these conditions, when they arise in a specific form of excitation delocalised over identical chromophores in a well-defined region $[21,22]$. Each component is necessarily positioned in an electronically equivalent local environment, and each is also coupled to its neighbours by transition dipole Coulombic interactions [23] of equal magnitude. A circularly symmetric ring array fulfills the basic criteria. Let the position of each specific emitter $(i=1,2,3)$ be labelled by cylindrical coordinates $\left(r_{i}, \phi_{i}, z_{i}\right)$, the array as a whole residing in the $\left(r_{i}, \phi_{i}\right.$, $0)$ plane. In principle, the individual emitters can be any species with a well-defined emission spectrum and an associated transition dipole with a source-fixed orientation. Although not essential, molecular fluorophores physically associated with nanoantennas can benefit from enhanced fluorescent emission, producing significantly increased optical output, potentially by several orders of magnitude [24-29]. An additional advantage of such a scheme is that the necessary coupling between neighbours can be supported over a larger distance.

The simplest configuration - one that also allows the possibility of identifying distinct left- and right-handed senses of twist in the output wave-front - is an array of three identical nanoemitters. This is the smallest number of emitters that can satisfy the necessary symmetry conditions: two emitters could generate a planar phase discontinuity, but not a vortex structure [30]. The three sources are positioned symmetrically in a ring, in this case forming an equilateral triangle. To satisfy the condition on the equivalence of every neighbouring pair interaction, it is also necessary that each emitter has an orientation rotated by $2 \pi / 3$ radians with respect to its neighbour, in either a clockwise or anticlockwise sense of rotation in the $(x, y)$ plane defined by the array locations in Cartesian form. In consequence, since the orientation of each source determines the angular disposition of its emission dipole moment, the projection of the three transition dipoles on the array plane is analogous to that of a three-spoke propeller, as seen in Figure 1. To accommodate the resulting threefold rotational symmetry, in a geometry that can also support the sought phase dissymmetry in the emission (necessarily precluding mirror symmetry perpendicular to the array plane), the array should conform to one of just two molecular point groups: those with Schoenflies designations $C_{3}$ and $C_{3 \mathrm{~h}}$. This rules out emitter dipoles whose projection on the plane has a simple radial disposition. The excitation of such a molecular
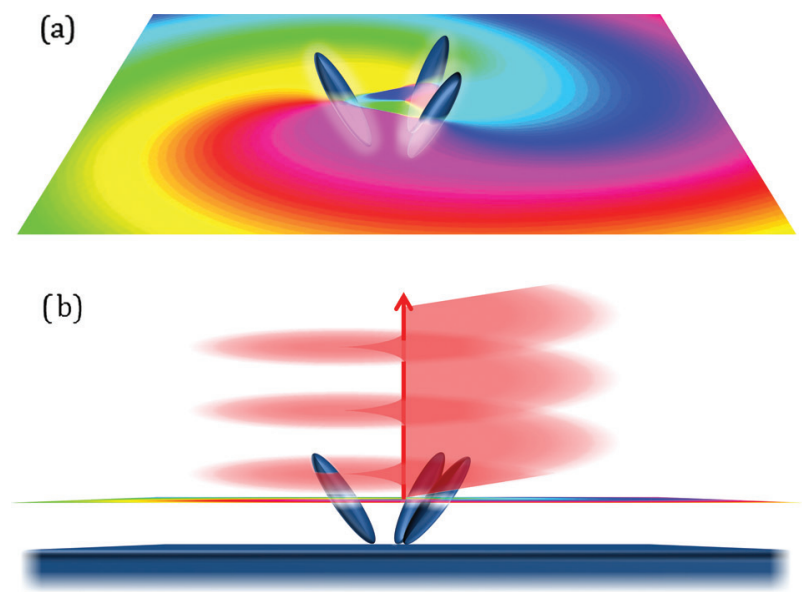

Figure 1 Chiral array structure of $C_{3}$ point group symmetry: (a) calculated exciton phase cross-section in the array plane; (b) side view showing chromophore support and vortex emission (artist's impression).

configuration to form an exciton may arise through various methods, not specifically limited to direct optical excitation; resonance energy transfer from a pre-excited neighbouring species in a higher electronic level is one obvious possibility $[31,32]$.

The threefold array can support excitons of two types, determined by the $A$ and $E$ irreducible representations of the point group $C_{3}$. In the case of transition dipoles lying in the $(x, y)$ plane, this arrangement corresponds to the $A^{\prime}$ and $E^{\prime}$ representations of the $C_{3 \mathrm{~h}}$ group [33]. It transpires that the doubly degenerate $E$ excitons provide the basis for the sought optical emission. Such degenerate excitons can be formed if there is a single or a double electronic excitation distributed amongst the three emitters; the former - an amount of delocalised energy associated with just one of the three emitters being in the excited state - is more readily achieved without the levels of population inversion requiring pulsed laser radiation, and this is the case we shall assume.

The Hamiltonian, $H$, for the system is based on the following $(3 \times 3)$-matrix:

$$
H=\left(\begin{array}{ccc}
E_{u} & \boldsymbol{\mu}_{1} \cdot \mathbf{V}_{12}\left(\mathbf{R}_{12}\right) \cdot \boldsymbol{\mu}_{2} & \boldsymbol{\mu}_{1} \cdot \mathbf{V}_{13}\left(\mathbf{R}_{13}\right) \cdot \boldsymbol{\mu}_{3} \\
\boldsymbol{\mu}_{2} \cdot \mathbf{V}_{21}\left(\mathbf{R}_{21}\right) \cdot \boldsymbol{\mu}_{1} & E_{u} & \boldsymbol{\mu}_{2} \cdot \mathbf{V}_{23}\left(\mathbf{R}_{23}\right) \cdot \boldsymbol{\mu}_{3} \\
\boldsymbol{\mu}_{3} \cdot \mathbf{V}_{31}\left(\mathbf{R}_{31}\right) \cdot \boldsymbol{\mu}_{1} & \boldsymbol{\mu}_{3} \cdot \mathbf{V}_{32}\left(\mathbf{R}_{32}\right) \cdot \boldsymbol{\mu}_{2} & E_{u}
\end{array}\right),
$$

where $E_{u}$ represents the energy of an isolated (unperturbed) molecule in its excited upper level $u$. The off-diagonal elements denote interactions between emitter pairs as signified by the subscripts, cast in the form of inner products of transition dipole moments $\boldsymbol{\mu}_{r}$ and $\boldsymbol{\mu}_{s}$ with a second-rank tensor $\mathbf{V}_{r s}$ (representing the coupling between the pair labeled $r$, $s$ ) whose familiar short-range form is detailed elsewhere [34]. This tensor has components that depend on the vector displacement of the relevant nanoemitters, e.g. $\mathbf{R}_{r s}=$ $\mathbf{R}_{r}-\mathbf{R}_{s}$. The orientational symmetry of the system results in each of the off-diagonal terms in (1) delivering the same 
Coulombic energy, $U$, given by;

$$
U=\mu_{r \mid i} V_{r s \mid i j}\left(\mathbf{R}_{r s}\right) \mu_{s \mid j}=\frac{\mu_{r \mid i} \mu_{s \mid j}}{4 \pi \varepsilon_{0} R_{r s}^{3}}\left(\delta_{i j}-3 \hat{R}_{r s \mid i} \hat{R}_{r s \mid j}\right),
$$

where indices $i, j$ signify summed Cartesian components, $\hat{R}_{r s}$ is the unit vector along $\mathbf{R}_{r s}$ and $R_{r s}$ is the corresponding distance such that $\mathbf{R}_{r s}=R_{r s} \hat{\mathbf{R}}_{r s}$. Setting the three transition dipole moments in an equidistant configuration and fixing their orientations, as defined previously, provides conditions suitable for the sought optical vortex creation: all off-diagonal elements of the matrix (1) return the same value, $U$.

Proceeding by diagonalising the matrix (1), we identify its eigenvectors as the coupled exciton wavefunctions. In terms of individual emitter basis functions $\xi_{0 / u}^{(i)}$ ( 0 for the ground state, $u$ for the electronically excited state) these wavefunctions are expressed as:

$$
\begin{aligned}
\left|\psi_{ \pm}^{\mathrm{E}}\right\rangle= & \frac{1}{\sqrt{3}}\left\{\left|\xi_{u}^{(1)} ; \xi_{0}^{(2)} ; \xi_{0}^{(3)}\right\rangle+\varepsilon\left|\xi_{0}^{(1)} ; \xi_{u}^{(2)} ; \xi_{0}^{(3)}\right\rangle\right. \\
& \left.+\varepsilon^{*}\left|\xi_{0}^{(1)} ; \xi_{0}^{(2)} ; \xi_{u}^{(3)}\right\rangle\right\},
\end{aligned}
$$

where $\varepsilon=\exp ( \pm 2 \pi i / 3)$, the sign correlating with the subscript index on the state wavefunction. The $E$ excitons, which exactly correspond with the sought distributions of phase about the symmetry axis, are separated in energy from the $A$ form by $3 U$ : this feature enables the selective excitation of one symmetry type [22]. The $E$ exciton distributions accordingly map into the optical field produced by their decay, leading to an optical vortex.

\section{Vortex emission}

The equations for the optical field produced by spontaneous decay of the excitons are developed by the same methods that have recently been described in the context of optical emission from a nanoemitter pair [35]. Relating to Eq. (3), the electric field at a general position $\mathbf{R}$ is now directly expressible as;

$$
\mathbf{E}_{123}(\mathbf{R})=-\sum_{r=1,2,3} \varepsilon^{r-1} \boldsymbol{\mu}_{r} . \mathbf{V}\left(\mathbf{R}_{r}\right)
$$

where $\mathbf{R}_{r}$ defines the location with respect to each nanoemitter $r$. Our interest focuses upon the development of the phase for the scalar optical field, which is secured as the complex argument $\theta(\mathbf{R})=\arg \left(\mathbf{E}_{123}(\mathbf{R})\right)$.

Figure 2 exhibits color-coded representations [36] of the phase of the scalar field at $z=0$, transverse to the output, and corresponding to the right-handed component of the pair; the axial phase discontinuity is characteristic of vortex structures. Emission perpendicular to the array will have an opposite sense of wave-front helicity for exciton emission in opposite directions; the pair interchange chiral character under mirror operation. In practice, the attachment of the array to a surface will favour outward emission. The degeneracy of the exciton pair supports the emission of photons in a quantum superposition state, simultaneously rotating in clockwise and anticlockwise directions. This type of superposition exactly corresponds to the optical field structures analysed in recent experiments by Zeilinger's group [37].

Despite the ubiquity of scalar field representations in connection with optical vortices, it is instructive to consider in more detail the vector character of the emission dipoles, and the optical polarisation. We start with the observation that the emitter transition dipoles cannot be normal to the array plane, for this would introduce mirror planes undermining the necessary symmetry. In fact, individual transition dipoles in the direction of the surface normal would in any case generate longitudinal fields, limited to the nearzone [38]. For the emission of an optical field that can be sustained in the propagating wave-zone, transverse fields are required.

Another consideration is resolving the degeneracy of the two $E$ states, to secure a single, specific handedness for the vortex emission. This will also serve to preclude the generation of any superposition state lacking the sought chirality. The solution is to remove the degeneracy between the $x$ and $y$ directions, as might be achieved with an anisotropic substrate. One possibility is a suitably chosen surface of a dielectric crystalline material; another is a lithographically ruled surface. There would also be a case for using uniaxial mechanical stress, as in the recent study of a cylindrically symmetric nanorod assembly, to achieve a similar lifting of degeneracy $[39,40]$. Note that the chiral nature of the array is necessary to produce the phase vortex; separating the two components of opposite handedness requires perturbing the symmetry, breaking the rotational symmetry.

\section{Discussion}

The discovery that it is possible to directly create optical vortex light from a radiation vacuum state invites a reconsideration of an earlier commentary on the subject. One of the first papers to formulate twisted beam interactions, in a fully quantised framework, stated that 'it is not possible for a system to spontaneously emit a twisted beam' [41]. That remark was made in the context of an analysis that separated both the radial amplitude and azimuthal phase functions from a quantised representation of the optical vortex states. The scheme that has been presented here departs from any such limitation by providing for a spatially distributed and phase-weighted form for spontaneous photon emission. In a sense, the exciton structures that are supported by the multiple emitter array might seem to act in a similar manner to a spiral wave-plate. However, it is to be emphasised that the mechanism we have reported specifically requires coupling between emitters that are physically separated, electronically distinct and individually anisotropic in their optical properties - which invites construction on a molecular basis. 

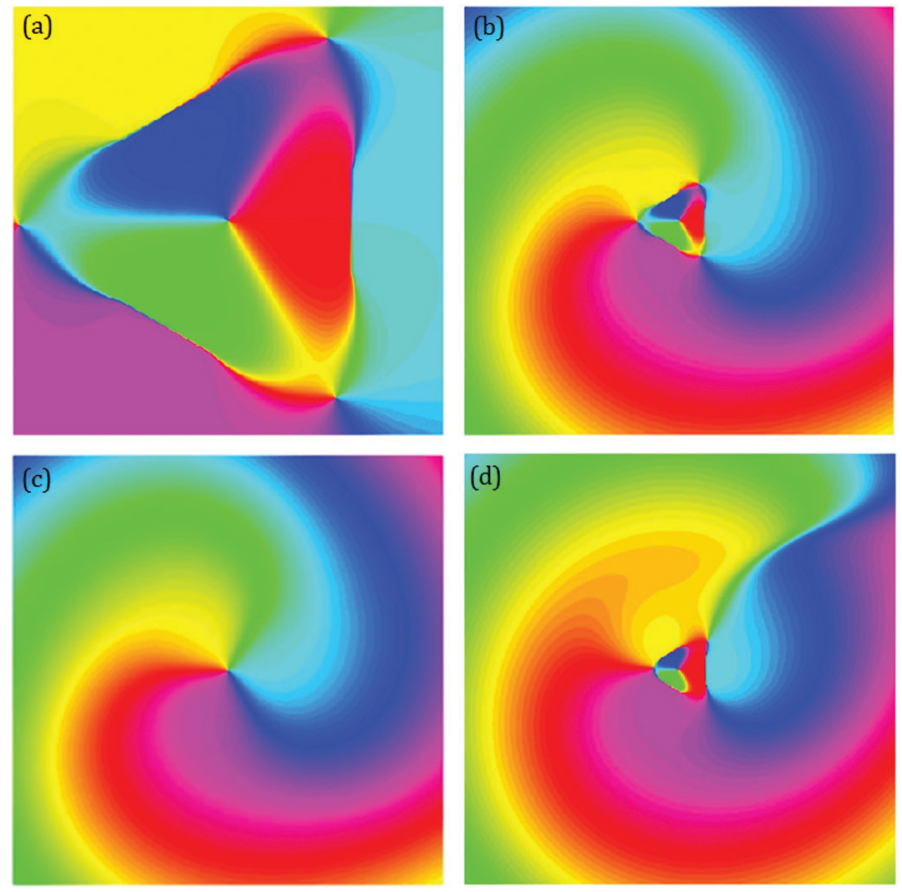

Figure 2 Phase diagrams of the scalar optical field for the three nanoemitters, in planes parallel to the array: (a) in the vicinity of the emitters, at $z=0$; (b) panoramic view at $z=$ 0 ; (c) in an infinitely displaced plane, parallel to the array; (d) phase of the $x$ field component corresponding to (b). Each antenna has an orientation rotated by $2 \pi / 3$ radians with respect to its neighbour and $\pi / 4$ radians with respect to the $z$-plane. The separation of the emitters is one tenth of the emission wavelength.

The degree of directed character, at least in the wavezone region, will be similar to that of a single emitter, in contrast to the collimated beam structure achieved by the more familiar methods converting conventional laser light. It is notable that the measurement of helical structure in even a single photon has proven tractable, using interferometric methods [18], but here there is more scope to explore possibilities for securing a specifically directed output, using stimulated emission, incorporating a cavity or a collection of arrays, with either a coplanar or vertically stacked architecture. Moreover, recent advances have proven the possibility of detecting and resolving discrete optical vortex states by coupling into an optical fibre [42]. It is hoped that the present analysis will pave the way for wide-ranging studies that will further develop the principle of optical vortex generation by launching from exciton states.

Acknowledgements. We gratefully acknowledge funding of this research by the Leverhulme Trust and the Engineering and Physical Sciences Research Council.

Received: 9 August 2013, Accepted: 12 September 2013

Published online: 18 October 2013

Key words: Optical vortex, Laguerre-Gaussian beam, twisted light, nanophotonics, orbital angular momentum, quantum optics.

\section{References}

[1] Y. Xu, X. Zhang, S. Dai, B. Fan, H. Ma, J.-1. Adam, J. Ren, and G. Chen, J. Phys. Chem. C 115, 13056-13062 (2011).

[2] I. Bialynicki-Birula and Z. Bialynicka-Birula, J. Opt. 13, 064014 (2011).
[3] H. Lin, D. Chen, Y. Yu, A. Yang, and Y. Wang, Opt. Lett. 36, 876-878 (2011).

[4] D. L. Andrews, Structured Light and its Applications: An Introduction to Phase-Structured Beams and Nanoscale Optical Forces (Academic, Amsterdam, Boston, 2008).

[5] J. P. Torres and L. Torner, Twisted Photons (Wiley, Berlin, 2011).

[6] A. M. Yao and M. J. Padgett, Adv. Opt. Photon. 3, 161-204 (2011).

[7] M. Padgett and R. Bowman, Nat. Photonics 5, 343-348 (2011).

[8] D. L. Andrews and M. Babiker, The Angular Momentum of Light (Cambridge University Press, Cambridge, 2012).

[9] D. L. Andrews, L. C. Dávila Romero, and M. Babiker, Opt. Commun. 237, 133-139 (2004).

[10] M. Babiker, C. R. Bennett, D. L. Andrews, and L. C. Dávila Romero, Phys. Rev. Lett. 89, 143601 (2002).

[11] V. E. Lembessis and M. Babiker, Phys. Rev. Lett. 110, 083002 (2013).

[12] D. L. Andrews, Phys. Rev. A 81, 033825 (2010).

[13] J. Leach, M. J. Padgett, S. M. Barnett, S. FrankeArnold, and J. Courtial, Phys. Rev. Lett. 88, 257901 (2002).

[14] G. Gibson, J. Courtial, M. J. Padgett, M. Vasnetsov, V. Pas'ko, S. M. Barnett, and S. Franke-Arnold, Opt. Express 12, 5448-5456 (2004).

[15] J. C. García-Escartín and P. Chamorro-Posada, Phys. Rev. A 78, 062320 (2008).

[16] C. J. Broadbent, P. Zerom, H. Shin, J. C. Howell, and R. W. Boyd, Phys. Rev. A 79, 033802 (2009).

[17] D. L. Andrews, Proc. SPIE 7754, 77540T (2010).

[18] E. J. Galvez, L. E. Coyle, E. Johnson, and B. J. Reschovsky, New J. Phys. 13, 053017 (2011).

[19] D. L. Andrews, Proc. SPIE 7950, 79500L (2011). 
[20] J. Wang, J.-Y. Yang, I. M. Fazal, N. Ahmed, Y. Yan, H. Huang, Y. Ren, Y. Yue, S. Dolinar, M. Tur, and A. E. Willner, Nat. Photonics 6, 488-496 (2012).

[21] H. van Amerongen, L. Valkūnas, and R. van Grondelle, Photosynthetic excitons (World Scientific Publishing, 2000).

[22] R. D. Jenkins and D. L. Andrews, J. Chem. Phys. 118, 34703479 (2003).

[23] L. Novotny and B. Hecht, Principles of Nano-Optics (Cambridge University Press, Cambridge, 2006).

[24] S. Kühn, U. Håkanson, L. Rogobete, and V. Sandoghdar, Phys. Rev. Lett. 97, 017402 (2006).

[25] R. M. Bakker, V. P. Drachev, Z. Liu, H.-K. Yuan, R. H. Pedersen, A. Boltasseva, J. Chen, J. Irudayaraj, A. V. Kildishev, and V. M. Shalaev, New J. Phys. 10, 125022 (2008).

[26] R. Filter, S. Mühlig, T. Eichelkraut, C. Rockstuhl, and F. Lederer, Phys. Rev. B 86, 035404 (2012).

[27] E. B. Ureña, M. P. Kreuzer, S. Itzhakov, H. Rigneault, R. Quidant, D. Oron, and J. Wenger, Adv. Mater. 24, OP314OP320 (2012).

[28] M. P. Busson, B. Rolly, B. Stout, N. Bonod, and S. Bidault, Nat. Commun. 3, 962 (2012).

[29] L. Novotny and N. van Hulst, Nat. Photonics 5, 83-90 (2011).

[30] J. S. Ford, D. S. Bradshaw, and D. L. Andrew, J. Phys. Chem. C, 12393-12396 (2013).
[31] D. L. Andrews and A. A. Demidov, Resonance Energy Transfer (Wiley, Chichester, 1999).

[32] D. L. Andrews, C. Curutchet, and G. D. Scholes, Laser \& Photon. Rev. 5, 114-123 (2011).

[33] J. A. Salthouse and M. J. Ware, Point Group Character Tables (Cambridge University Press, Cambridge, 1972).

[34] D. S. Bradshaw and D. L. Andrews, J. Phys. Chem. A 117, 75-82 (2013).

[35] E. M. Rice and D. L. Andrews, J. Chem. Phys. 136, 244503 (2012).

[36] J. Leach, M. R. Dennis, J. Courtial, and M. J. Padgett, New J. Phys. 7, 55 (2005).

[37] R. Fickler, R. Lapkiewicz, W. N. Plick, M. Krenn, C. Schaeff, S. Ramelow, and A. Zeilinger, Science 338, 640-643 (2012).

[38] D. L. Andrews and D. S. Bradshaw, Eur. J. Phys. 25, 845-858 (2004).

[39] S. D. Liu, Z. Yang, R. P. Liu, and X. Y. Li, ACS Nano 6, 6260-6271 (2012).

[40] Y. H. Cui, J. H. Zhou, V. A. Tamma, and W. Park, ACS Nano 6, 2385-2393 (2012).

[41] L. C. Dávila Romero, D. L. Andrews, and M. Babiker, J. Opt. B: Quantum Semiclass. Opt. 4, S66 (2002).

[42] D. A. Nolan, G. Milione, and R. R. Alfano, Proc. SPIE 8637, 86370E (2013). 